\title{
Contribution of FPR and TLR9 to hypoxia-induced chemoresistance of ovarian cancer cells
}

This article was published in the following Dove Medical Press journal: OncoTargets and Therapy

\author{
Yongqing $\mathrm{Cai}^{1}$ \\ Jian Huang ${ }^{2}$ \\ Haiyan Xing ${ }^{\prime}$ \\ Bin $\mathrm{Li}^{\prime}$ \\ Ling $\mathrm{Li}^{\prime}$ \\ Xianfeng Wang' \\ Dan Peng' \\ Jianhong Chen' \\ 'Department of Pharmacy, Daping \\ Hospital and Research Institute of \\ Surgery, Army Medical University, \\ Chongqing 400042, China; \\ ${ }^{2}$ Department of High Altitude Biology \\ and Pathology, High Altitude Military \\ Medical College, Army Medical \\ University, Chongqing 400042, China
}

Correspondence: Jianhong Chen Department of Pharmacy, Daping Hospital and Research Institute of Surgery, Army Medical University, No 10 Changjiang Road, Yuzhong District, Chongqing 400042, China Tel +860236875 709l Email chenjh-110@263.net
Background/purpose: The aim of this study was to investigate the role and mechanisms of the formyl peptide receptor (FPR) and the toll-like receptor 9 (TLR9) in hypoxia-induced chemoresistance of human ovarian cancer cells.

Materials and methods: SKOV3 cells were exposed to hypoxia for 24 hours, the supernatant was collected to stimulate normoxia-cultured SKOV3, and the inhibition rate of cell growth was detected with CCK8 test. The agonist of TLR9 CpG ODN and the agonist of FPR FMLF were applied to investigate the chemosensitivity of SKOV3 cells to cisplatin. The cells were also treated with FPR antagonist t-Boc or TLR9 antagonist CQ. Western blot was applied to detect protein levels of FPR, TLR9, MRP, P-gp, p53 and Beclin-1. Immunofluorescence staining was applied to observe the distribution of TLR9 in SKOV3 cells.

Results: Hypoxia exposure reduced the inhibition rate of cisplatin on SKOV3 cells. WB showed that FPR and TLR9 were expressed in human ovarian cancer tissues and SKOV3 cells, and the levels were increased with longer hypoxia time. After SKOV3 was stimulated with fMLF or ODN2006, cisplatin-induced inhibition rate was significantly decreased. tBoc and CQ significantly attenuated hypoxia supernatant-induced chemoresistance of SKOV3 cells. Hypoxia supernatants significantly increased MRP, P-gp, p53 and Beclin-1 proteins in SKOV3 cells, which were significantly reduced by tBoc.

Conclusion: Hypoxia upregulates the expression of FPR and TLR9, and promotes the release of ligands for both receptors in human ovarian cancer cell line. FPR and TLR9 may be noval targets for chemosensitizing to ovarian cancer cells.

Keywords: human ovarian cancer, hypoxia, chemoresistance, FPR, TLR9, MRP, Beclin-1, HICR

\section{Introduction}

Chemoresistance of cancer cells remains a challenge for chemotherapy of cancer patients. Therefore, it is an important to understand the mechanisms of drug resistance. Gene mutations or amplifications in cancer cells that influence the uptake, metabolism or export of drugs were considered to contribute to chemotherapy resistance. Specific microenvironment in tumors, such as hypoxic conditions, also increases drug efflux and plays an important role in chemoresistance. Hypoxia is a common feature of rapidly growing tumors, and promotes cancer cell resistance to apoptosis. ${ }^{1,2}$

Under hypoxic conditions, a number of chemical substances are released, such as HMGB1 and mitochondrial DNA, activating toll-like receptor (TLR) thus inducing various pathophysiological responses including high-altitude adaptation, wound healing, atherosclerosis and tumorigenesis. ${ }^{3}$ TLR9 is a pattern recognition receptor and is capable of activating myeloid differentiation factor 88-dependent pathway, leading to activation of NF- $\kappa \mathrm{B}$ and mitogen-activated protein kinases (MAPKs) including c-Jun $\mathrm{N}$-terminal kinase and $\mathrm{p} 38$, which then induce the release of NO and pro-inflammatory 
cytokines. $^{4}$ The most potent activator of angiogenesis in tumors is hypoxia. Activated TLR9 up-regulates inhibitor of apoptosis such as Bcl-xL, cFLIP and survivin, reducing the chemosensitivity of cancer cells. ${ }^{5}$

The formyl peptide receptor (FPR) belongs to a class of $G$ protein-coupled receptors involved in chemotaxis. FPR was originally identified by its ability to bind $\mathrm{N}$-formyl peptides such as $\mathrm{N}$-formylmethionine produced by the degradation of either bacterial or host cells. FPR is involved in mediating immune cell response to infection. It may also act to suppress the immune system under certain conditions. Impaired mitochondria release methylated peptides to activate FPR receptor-mediated signaling pathway. FPR promotes the malignant behaviors of tumor cells, ${ }^{6-8}$ but its role in drug resistance is not clear. In this study, we investigate whether FPR/TLR9 are capable of promoting chemotherapy resistance in ovarian cancer cells.

\section{Materials and methods Reagents}

Cisplatin, chloroquine (CQ), tertiary butyl formyl oxygen (tBoc) and N-formylmethionyl-leucyl-phenylalanine (fMLF) were purchased from Sigma (Sigma-Aldrich Co., St Louis, MO, USA). TLR9, multidrug resistance-associated protein (MRP), FPR, Beclin-1, p53 and P-gp antibodies were purchased from Abcam (Cambridge, UK). Monkey anti-rat and anti-rabbit secondary antibodies were purchased from Thermo Scientific (Thermo Fisher Scientific, Waltham, MA, USA). Streptomycin sulfate solution, Cell Lysis buffer, BCA protein assay kit, BeyoECL plus Kit, goat anti-rabbit and anti-rat secondary antibody were purchased from Beyotime (Shanghai, China).

All primers were synthesized by Invitrogen Ltd. Co. (Thermo Fisher Scientific). All oligodeoxynucleotides used in the experiments were in phosphorothioate backbone. All oligodeoxynucleotides were dissolved and diluted with phosphate-buffered saline (0.01 M PBS, pH 7.4).

\section{Cell culture}

Human ovarian cancer cell line (SKOV3) was purchased from American Type Culture Collection (ATCC, Manassas, VA, USA). Ovarian cancer tissues were provided by the department oncology of Daping Hospital of Army Medical University (Chongqing, China). The samples of cancer tissues (Histological type: serous, FIGO stage: III) from nine chemonaïve patients were analyzed in this study. The study was approved by the Ethics Committee of Daping Hospital of Army Medical University. The patient consent was written informed consent, and this was conducted in accordance with the Declaration of Helsinki. The cells were cultured in 1640 culture medium (Thermo Fisher Scientific) supplemented with 10\% fetal bovine serum (Thermo Fisher Scientific) and antibiotics (100 U/ $\mathrm{mL}$ penicillin and $100 \mu \mathrm{g} / \mathrm{mL}$ streptomycin) in a $5 \% \mathrm{CO}_{2}$ atmosphere at $37^{\circ} \mathrm{C}$. Endotoxin levels in cell culture media and supernatants were undetectable $(<1 \mathrm{ng} / \mathrm{mg})$ as assessed by Limulus assay.

\section{Analysis of cell proliferation}

SKOV3 cells $\left(5 \times 10^{4} / \mathrm{mL}\right)$ were seeded in $96-$ well plates and treated with CpG ODN2006 (50 $\mu \mathrm{g} / \mathrm{mL}, \mathrm{CpG}-\mathrm{ODN})$ and CpG ODN 2006 control (50 $\mu \mathrm{g} / \mathrm{mL}, \mathrm{C}-\mathrm{ODN})$ for 24 hours and then treated with cisplatin $(3.5 \mu \mathrm{g} / \mathrm{mL})$. After incubation for another 24 hours, cells were incubated in 10\% CCK-8 that was diluted in normal culture medium at $37^{\circ} \mathrm{C}$ for 3 hours. The OD was read at $450 \mathrm{~nm}$ using a 96-well plate reader and the inhibition rate was calculated according to the following formula: $\left[\mathrm{OD}_{(\text {Blank })}-\mathrm{OD}_{(\mathrm{CpG}-\mathrm{ODN})}\right] /\left[\mathrm{OD}_{\text {(Blank) }}-\right.$ $\left.\mathrm{OD}_{(\mathrm{C}-\mathrm{ODN})}\right] \times 100 \%$.

SKOV3 cells $\left(5 \times 10^{4} / \mathrm{mL}\right)$ were seeded in 96 -well plates and treated with fMLF (100 nM), tBoc (100 nM) and fMLF $(100 \mathrm{nM})+$ tBoc $(100 \mathrm{nM})$ for 6 hours, 12 hours and 24 hours, then treated with cisplatin $(3.5 \mu \mathrm{g} / \mathrm{mL})$. After incubation for another 24 hours, cells were incubated in $10 \%$ CCK-8 that was diluted in normal culture medium at $37^{\circ} \mathrm{C}$ for 3 hours. The inhibition rate was calculated as described above.

SKOV3 cells were divided into hypoxia group $\left(1 \% \mathrm{O}_{2}\right)$ and normoxia group $\left(21 \% \mathrm{O}_{2}\right)$ and incubated for 12 hours. The supernatant was collected and diluted with fresh medium to volume ratio concentration of $0 \%, 40 \%, 60 \%$, $80 \%$ and $100 \%$. SKOV3 cells was incubated with these supernatant for 24 hours and then treated with cisplatin $(3.5 \mu \mathrm{g} / \mathrm{mL})$. The inhibition rate was calculated as described above. The hypoxia-induced chemoresistance (HICR) was calculated by the formula: $\operatorname{HICR}(\%)=\left(\operatorname{IR}_{\text {normoxia }}-\operatorname{IR}_{\text {hypoxia }}\right) /$ $\mathrm{IR}_{\text {hypoxia }} \times 100 \%$.

SKOV3 cells were pretreated with concentrations of tBoc or CQ $(0,1,10,100,1,000 \mathrm{nM})$ for 3 hours, then the supernatant was collected and diluted with fresh medium to volume ratio concentration of $80 \%$. The hypoxia group and normoxia group cells were incubated with these supernatant for 24 hours and then treated with cisplatin 
(3.5 $\mu \mathrm{g} / \mathrm{mL})$. The inhibition rate and HICR were calculated as described above.

\section{Western blot}

Total cell protein was extracted and the concentration was measured with BCA protein assay kit. Subsequently, the protein was separated by $10 \%$ SDS-polyacrylamide gel electrophoresis, and transferred to PVDF membranes (Merck Millipore, Billerica, MA, USA). After blocking with skim milk in TBST (10 mM Tris- $\mathrm{HCl}, 0.1 \mathrm{M} \mathrm{NaCl}, 0.1 \%$ TWEEN 20, pH 7.4), membranes were incubated with antibodies diluted in 5\% skim milk, and then incubated with HRP conjugated secondary antibodies. Immunoreactive bands were visualized by ECL detection reagents, and then immunoblotting signals were scanned and quantitated using a ChemiDoc XRS gel imaging system (Bio-Rad Laboratories Inc., Hercules, CA, USA) and quantity one software, respectively. $\beta$-Actin served as the internal control (Beyotime).

\section{Real-time PCR}

Total RNA was extracted from human ovarian tissues using Total RNA kit I (Omega Bio-Tek, Doraville, GA, USA). Equal amounts $(2.0 \mu \mathrm{g})$ of total RNA were reversetranscribed to cDNA by using the PrimeScript RT reagent kit (Takara, Dalian, China) for use in PCR. TLR9 mRNA levels were measured by real-time PCR using SYBR Premix Ex Taq II (Takara, Dalian, China) and normalized to $\beta$-actin. The following primers synthesized by Sangon Biotech (Shanghai, China) were used for PCR experiments: TLR9, 5'-TGTAATAACAGTTGCCGTCCAT-3' (forward) and $5^{\prime}$-CAGCCTTTCCTTGTCCTCC-3' (reverse); and $\beta$-actin, $5^{\prime}$-GTGGGGCGCCCAGGCACCT-3' (forward) and 5'-CTTCCTTAATGTCACACACGATTG-3' (reverse). The total mix was run on a 7900 real time system (Applied Biosystems by Life Technologies, Thermo Fisher Scientific) with the PCR conditions as follow: $95^{\circ} \mathrm{C}$ (30 seconds), $60^{\circ} \mathrm{C}(30$ seconds) and $72^{\circ} \mathrm{C}$ (30 seconds) for 40 cycles. The data are presented as the relative mRNA level normalized to $\beta$-actin and then expressed as the fold increase relative to the control.

\section{Immunofluorescence assay}

The cells were digested and inoculated in cell climbing piece for 24 hours, then washed by PBS and fixed by $4 \%$ polyphosphate. The sections were permeabilized with Triton X-100 $0.2 \%$ in PBS for 20 minutes, followed by incubation with blocking solution of serum at room temperature for 1 hour. After washing, the slides were incubated at $4^{\circ} \mathrm{C}$ overnight with TLR9 antibody (1:200). Then the sections were incubated with FITC-labeled secondary antibody for 1 hour, and stained with 4,6-diamidino-2-phenylindole (Thermo Fisher Scientific) for 15 minutes, and mounted in glycerol. Controls were treated by omitting the primary antibody. Sections were examined with a confocal laser scanning microscope.

\section{Statistical analysis}

All results were shown as the mean \pm SD. Data were pooled from at least three independent experiments, and analyzed by Student's $t$-test or ANOVA. $P$-values $<0.05$ were considered as statistically significant.

\section{Results \\ Hypoxia promotes chemoresistance of ovarian cancer cells}

To determine whether the chemosensitivity of ovarian cancer cells were affected by hypoxia, cell growth inhibition rates and HICR value of cisplatin on SKOV3 were measured after stimulation of hypoxia $\left(1 \% \mathrm{O}_{2}\right)$ for 6,12 and 24 hours. Only inhibition rates at 12 hours and 24 hours were significantly decreased compared with normoxia group $(P<0.01$, Figure 1A). Also only HICR at 12 hours (4.85) and 24 hours (8.66) were significantly increased compared with normoxia group $(P<0.01$, Figure 1B).

\section{Hypoxic cell supernatant induces chemoresistance of ovarian cancer cells}

To investigate whether the chemoresistance was affected by substances released from hypoxic ovarian cancer cells, the inhibition rates of cisplatin were measured in cells stimulated with supernatant from cells culture in hypoxia treatment for 6 hours, 12 hours and 24 hours. Concentrations of supernatant from cell with hypoxia treatment for 12 hours and 24 hours showed negative correlation with the inhibition rates of cisplatin. The difference between the normoxia group and the hypoxia group $(60 \%, 80 \%$ and $100 \%$ concentration of supernatant) was statistically significant. However, under hypoxia treatment for 6 hours, only inhibition rates of $100 \%$ group were decreased significantly compared to normoxia group (Figure 1C).

\section{The expression of FPR in ovarian cancer}

To identify the expression of FPR in ovarian cancer tissue, the FPR polyclonal antibody (recognizing three FPRs) was used. Five specimens of ovarian cancer expressed FPR at different levels (Figure 2A). We also detected the level of 

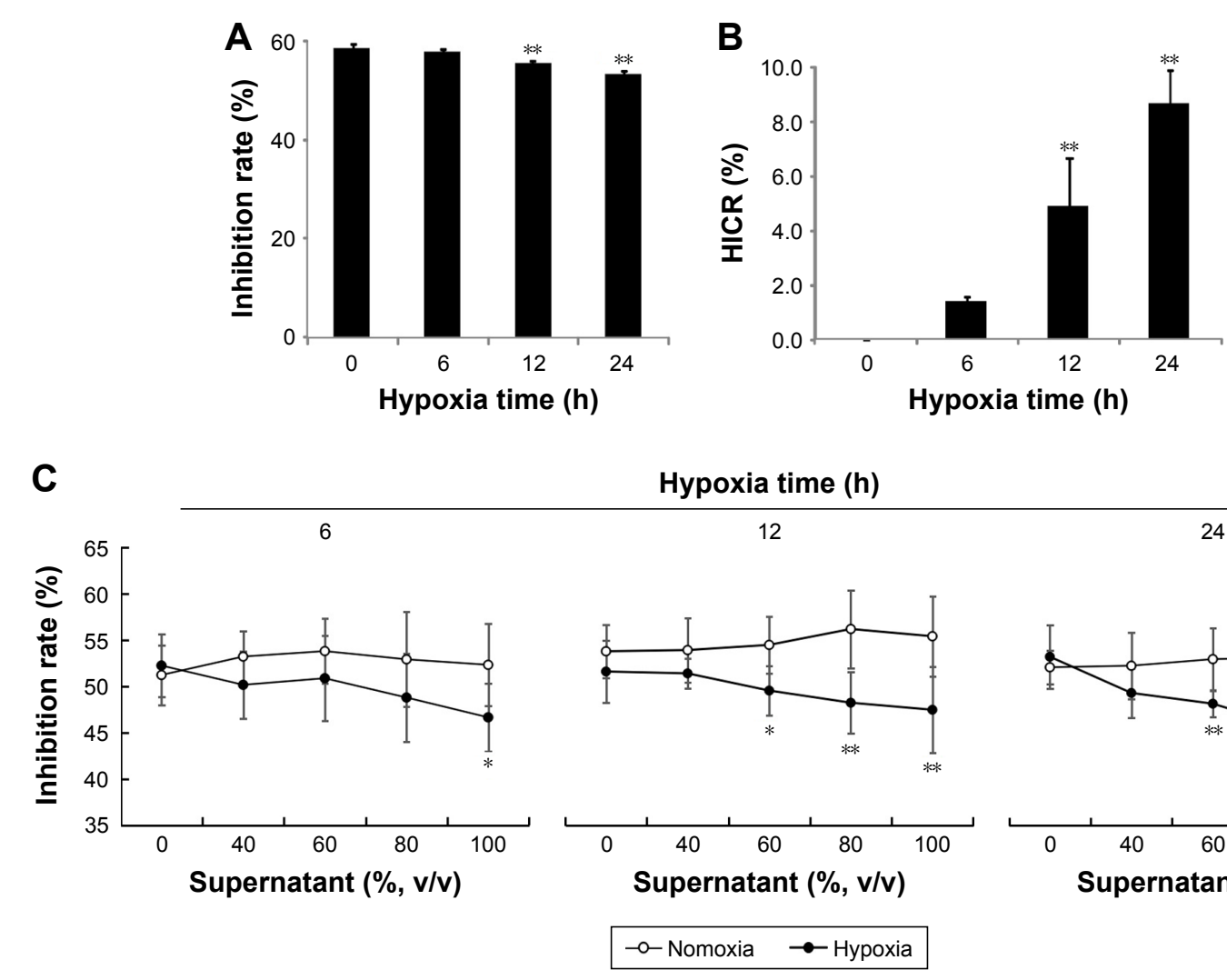

Hypoxia time (h)

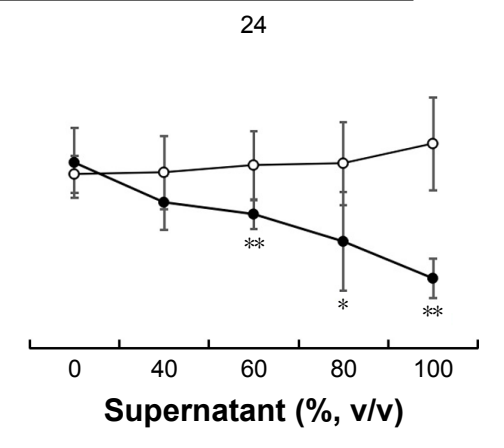

Figure I Hypoxia reduced the sensitivity of SKOV3 cells to cisplatin (mean \pm SD, $n=6, \%$ ).

Notes: SKOV3 cells were exposed in hypoxia ( $1 \% \mathrm{O}_{2}$, closed line) or normoxia (opened line) chamber, the supernatant were collected to stimulate another cells in normoxia for 24 hours. Then the cells were treated with cisplatin $(3 \mu \mathrm{g} / \mathrm{mL})$ for 24 hours, the viability was measured using CCK 8 assay kit by fluorescence microplate reader and inhibitive rate of cisplatin was calculated. (A) The inhibition rates of cisplatin to SKOV3 in hypoxia; (B) the HICR value of cisplatin to SKOV3 in hypoxia; (C) the inhibition rates of cisplatin to SKOV3 after treated with supernatant from hypoxia SKOV3 cells for different times. $* P<0.05$, $* * P<0.0$ I, compare with the normoxia SKOV3 cells at the same time and the same volume supernatant.

Abbreviations: HICR, hypoxia-induced chemoresistance; v/v, volume per volume.

MRP which reflects the drug resistance of the specimen. The level of MRP was higher in specimens with higher expression level of FPR, which was positively correlated with the levels of MRP ( $r=0.942$, Figure 2B). These results suggest that FPR may play an important role in the drug resistance of ovarian cancer cells.

To detect the function of FPR in ovarian cancer cells, the chemotactic experiment was performed. fMLF is the prototypical representative of the $\mathrm{N}$-fomylated oligopeptide family of chemotactic factors. It helps define the stimulus-response coupling mechanisms by which diverse chemotactic factors and the $\mathrm{G}$ protein-coupled receptors induce cellular function. Concentration of fMLF at $10,10^{2}, 10^{3} \mathrm{nM}$, cell chemotaxis increased with a bell-shaped curve migration (Figure 2C). Cells were inhibited by an FPR antagonist (tBoc), and the difference was statistically significant compared with the group without tBoc (Figure 2C).

Western blot was used to evaluate the expression of FPR in SKOV3 cells with normoxic and hypoxic conditions. FPR was expressed in ovarian cancer cells with both conditions. The level of FPR was in positive correlation with hypoxia time. The level of FPR at 12 hours and 24 hours statistically increased compared to normoxia cell group (Figure 2D).

\section{The effect of FPR on chemoresistance induced by hypoxia}

To determine how FPR regulates the chemosensitivity of ovarian cancer cells, HICR of cisplatin was detected with tBoc pretreatment cell receiving hypoxia stimulation for 6 hours, 12 hours and 24 hours. Inhibition rates were measured with fMLF pretreatment of cell receiving hypoxia stimulation for 6 hours, 12 hours and 24 hours. The results showed that cell proliferation inhibited by hypoxia was restored by tBoc pretreatment with reduced HICRs. At $10 \mathrm{nM}, 100 \mathrm{nM}$ and 1,000 nM tBoc, HICRs of hypoxia stimulation for 6 hours and 12 hours were significantly reduced. Pretreatment with $100 \mathrm{nM}$ tBoc followed by hypoxia stimulation for 24 hours, HICRs were significantly reduced (Figure 3A). 
A

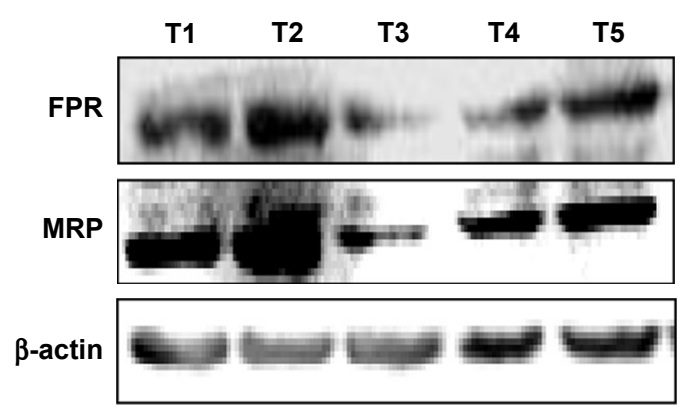

C

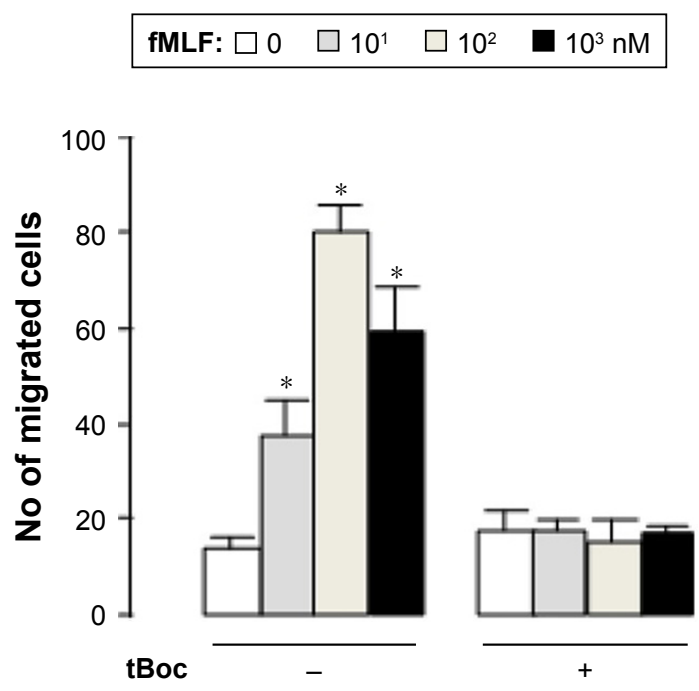

B

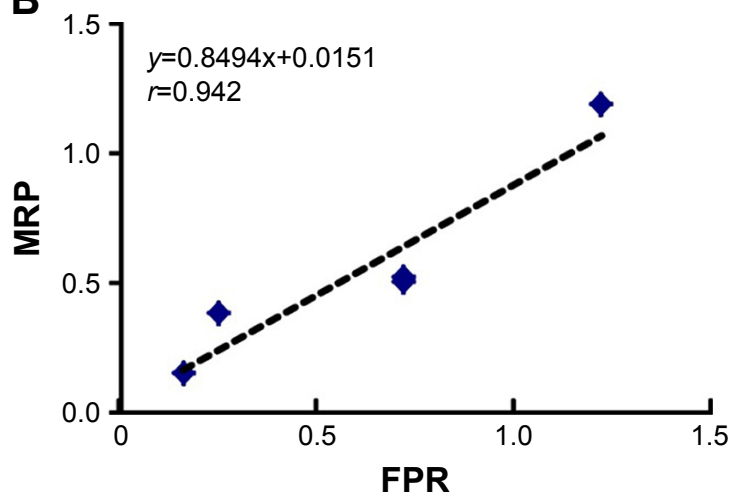

D

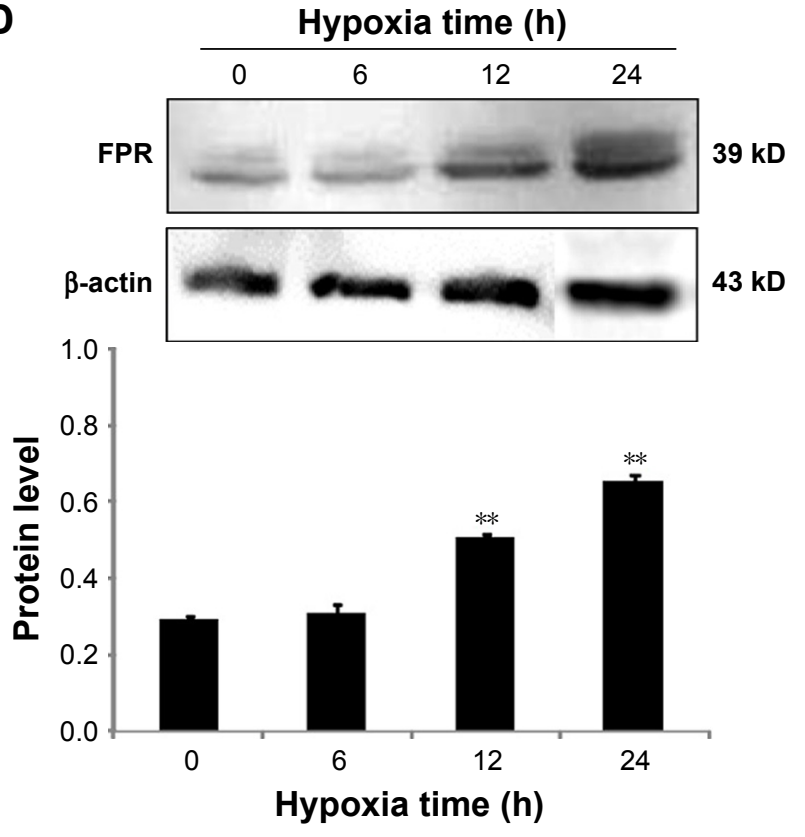

Figure 2 The expression of FPR in human ovarian cancer tissues.

Notes: (A) The expression levels of FPR and MRP in five ovarian cancer tissues; (B) relevance between FPR and MRP levels in human ovarian cancer tissues; (C) FPR mediated SKOV3 chemotaxis; (D) the protein level of FPR in SKOV3 cell under hypoxia. $* P<0.05$, compare with 0 nM fMLF; $* * P<0.05$, compare with normoxia group ( 0 hour). Abbreviations: FPR, formyl peptide receptor; MRP, multidrug resistance-associated protein; fMLF, N-formylmethionyl-leucyl-phenylalanine; tBoc, tertiary butyl formyl oxygen.

Our data demonstrate that the inhibition rates were increased and the chemoresistance was improved with stimulation of FPR agonist fMLF. This effect was reduced by tBoc pretreatment (Figure 3B).

\section{The role for FPR in hypoxia-induced expression of MRP, P-gp, p53 and Beclin-I} To investigate the mechanisms of FPR in HICR, the expression of MRP, P-gp, p53 and Beclin-1 in ovarian cancer cells were detected by Western blot in the presence or absence of tBoc pretreatment. The expression of MRP of hypoxia group was significantly upregulated compared to normoxia cell group; however, tBoc pretreatment downregulated expression of MRP (Figure 4). Similar results were shown for P-gp, p53 and Beclin-1 (Figure 4).

\section{The expression of TLR9 in ovarian cancer cells}

TLR9 was detected by WB. Nine specimens of ovarian cancer all expressed TLR9 (Figure 5A). The level of MRP was higher in specimens with higher expression level of TLR9. The expression level of TLR9 in ovarian cancer tissues was positively correlated with MRP ( $r=0.804$, Figure $5 \mathrm{~B})$. This result suggests that TLR9 may play an important role in the drug resistance of ovarian cancer cells. 


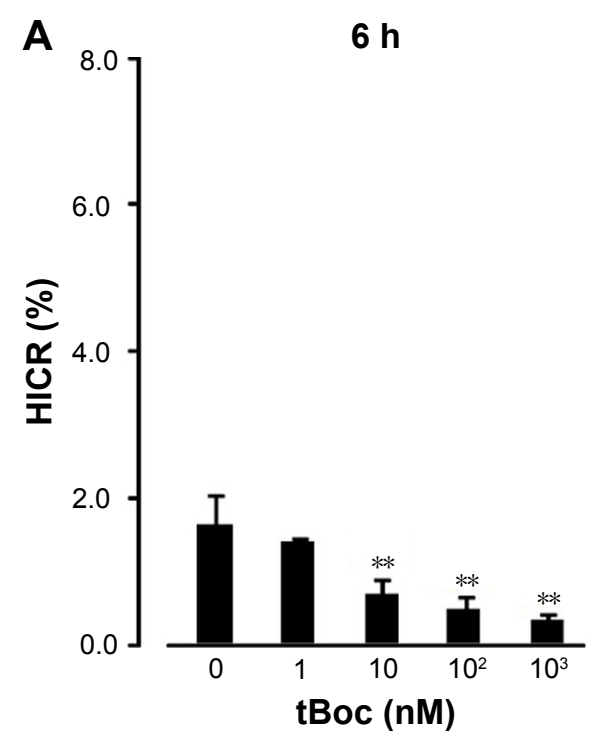

$12 \mathrm{~h}$

$24 \mathrm{~h}$
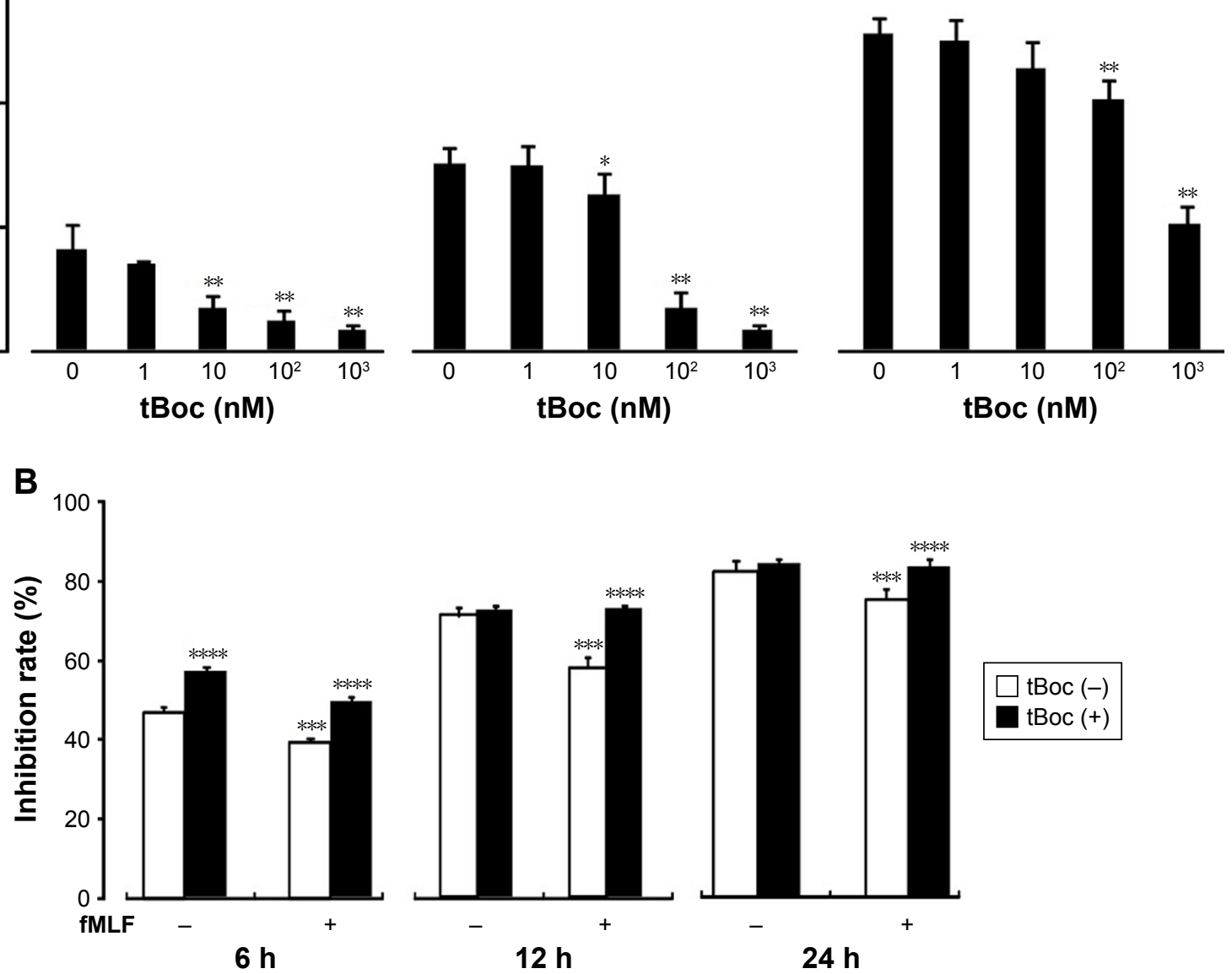

Figure 3 Effect of agonist and antagonist of FPR on the chemoresistance of SKOV3 cells to cisplatin treated with hypoxia supernatant.

Notes: (A) The chemoresistance of SKOV3 cells treated with FPR antagonist tBoc in hypoxia supernatant; (B) FPR activation promotes SKOV3 drug resistance (normoxia). $* P<0.05$, $* * P<0.01$, compare to group without $\mathrm{tBoc}$ at the same time; $* * * P<0.05$, compare with $\mathrm{fMLF}(-)$ group; $* * * * P<0.05$, compare with tBoc $(-)$ group.

Abbreviations: HICR, hypoxia-induced chemoresistance; tBoc, tertiary butyl formyl oxygen; fMLF, N-formylmethionyl-leucyl-phenylalanine; FPR, formyl peptide receptor.

Immunofluorescence was used to evaluate the expression of TLR9 in SKOV3 cells. TLR9 was expressed not only on cell membrane but also in cytoplasm of SKOV3 cells (Figure 5C). Under the hypoxic conditions, the level of TLR 9 at 12 hours and 24 hours was significantly upregulated compared to normoxia cell group (Figure 5D and E).

\section{The effect of TLR9 on SKOV3 cell}

\section{chemoresistance induced by hypoxia}

To determine whether the chemosensitivity of ovarian cancer cells to cisplatin was regulated by TLR9, the cell growth inhibition rates were measured after stimulation of the cells with a CpG ODN2006. After 6 hours, 12 hours and 24 hours, the relative inhibition rates of $\mathrm{CpG}-\mathrm{ODN}$ treated with group significantly decreased by $9.2 \%, 8.9 \%$ and $10.64 \%$, respectively, compared to C-ODN cell group with the $\mathrm{CpG}$ ODN pretreatment (Figure 6A).

To determine how TLR9 regulated the chemosensitivity of ovarian cancer cells, HICR of cisplatin was detected in cells with CQ pretreatment and hypoxia stimulation for 6 hours, 12 hours and 24 hours. Cell proliferation inhibited by hypoxia was restored by CQ pretreatment. With $10 \mu \mathrm{M}$ and $100 \mu \mathrm{M}$ CQ pretreatment, after hypoxia stimulation for 12 hours, the average reduction rate of inhibition rates was 2.07 and 1.46 times of that in normoxia cell group. Without CQ pretreatment, the average reduction rate was 3.92 times of normoxia cell group. With $10 \mu \mathrm{M}$ and $100 \mu \mathrm{M}$ of CQ after hypoxia stimulation for 24 hours, the HICR was 2.04 and 1.86; however, without CQ, the HICR was 5.17. The difference was statistically significant. With hypoxia stimulation 


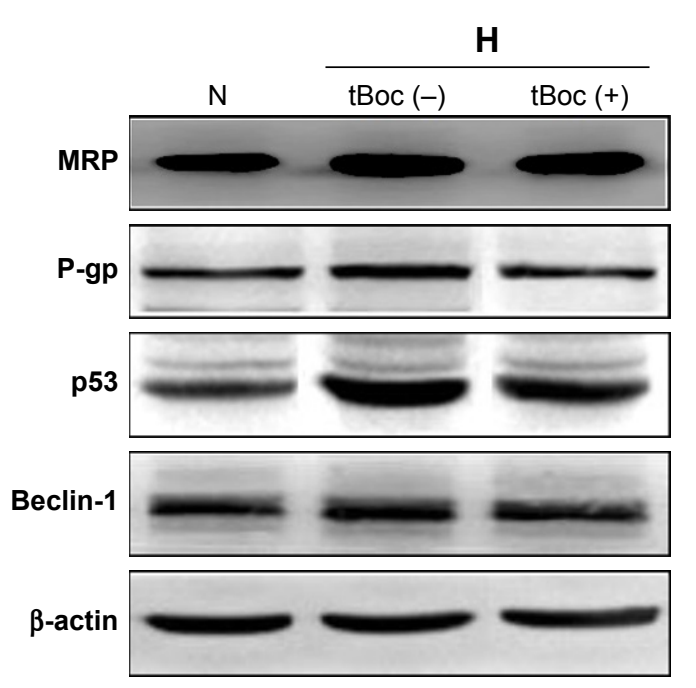

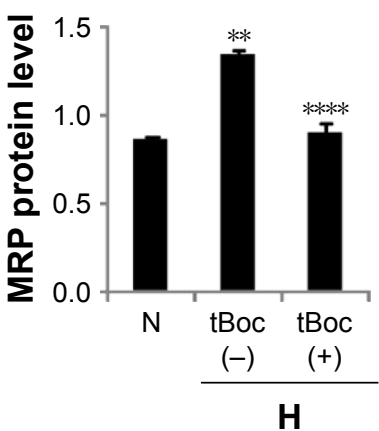

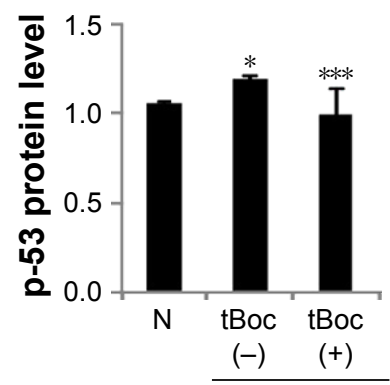

H
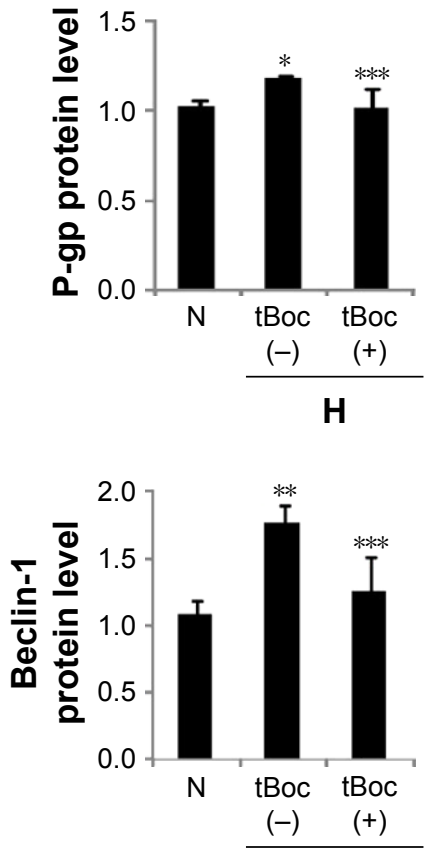

H

Figure 4 The expression of MRP, P-gp, p53 and Beclin-I in ovarian cancer cells with/without tBoc pretreatment.

Notes: $* P<0.05, * * P<0.0$ I, compared with normoxia cell group; $* * * P<0.05$, $* * * * P<0.01$, compare with hypoxia without tBoc cell group.

Abbreviations: MRP, multidrug resistance-associated protein; tBoc, tertiary butyl formyl oxygen.

for 6 hours, only HICR of $10 \mu \mathrm{M}$ CQ cell group (0.37) was statistically decreased compared to without CQ cell group (1.52). These results demonstrate that pretreatment with CQ enabled recovery of the chemosensitivity of ovarian cancer cells to cisplatin (Figure 6B).

\section{The role for TLR9 in hypoxia-induced expression of MRP, P-gp, p53 and Beclin-I} To investigate the mechanism of TLR9 in HICR, the expression of MRP, P-gp, p53 and Beclin-1 was detected by Western blot with CQ pretreatment. Our results showed that the expression of MRP in hypoxia cell group was significantly upregulated compared to normoxia cell group; however, the upregulated expression of MRP in cells with CQ pretreatment was significantly inhibited compared to hypoxia but without CQ cell group. Similar results were shown in studies of P-gp and $\mathrm{p} 53$. The expression of Beclin-1 in hypoxia cell group was upregulated compared to normoxia cell group, but there was no significant difference between CQ cell group and hypoxia without CQ cell group (Figure 7).

\section{Discussion}

Recent studies have shown the intracellular translocation and release of a variety of damage-associated molecular patterns (DAMPs) such as HMGB1 and mtDNA and formyl peptides that play a key role in hypoxia-induced tumor growth and chemotherapy resistance. This may be closely connected with activated TLR9 and FPR signal path way in hepatocellular cancer cells. ${ }^{9,10}$ Release of HMGB1 and activated TLR9-dependent pathways in cancer cells promote their adhesion, proliferation, migration and invasion thus increasing chemoresistance. ${ }^{5,11}$ Moreover, HIF-1 confers selective protection of tumor cell from apoptosis via induction of a truncated but active form of the voltagedependent anion channel 1, and allows cell survival in hypoxia with staurosporine and etoposide treatment. ${ }^{12}$ Our data showed the concentrations of supernatant from cancer cells with hypoxia treatment were positively correlated with cell chemoresistance, suggesting that release of DAMPs induced by hypoxia was triggered by attenuated chemosensitivity in ovarian cancer cells. FPR and TLR9 signal activated by DAMPs plays an important role in tumor progression.

Our study revealed that TLR9 was expressed both on the membrane and in the cytoplasm of ovarian cancer cells. Similar results were reported in which full-length TLR9 was expressed on the cell membrane rather than in the cytoplasm of hepatocellular cancer cells, whereas multiple cleaved forms of TLR9 were expressed in the cytoplasm. 
A

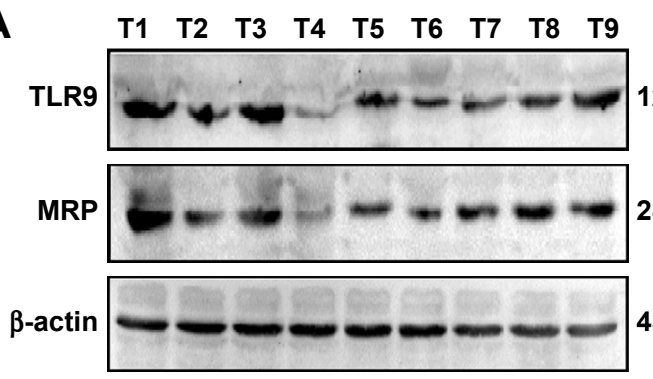

B

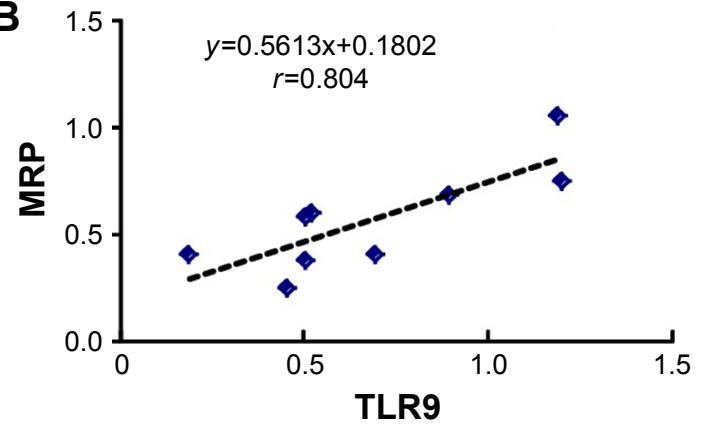

D

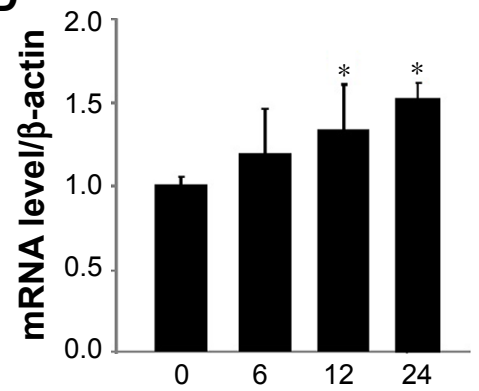

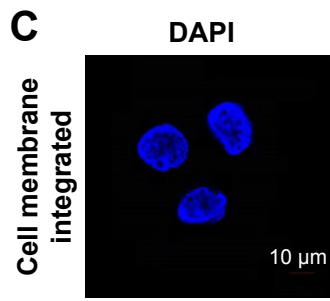
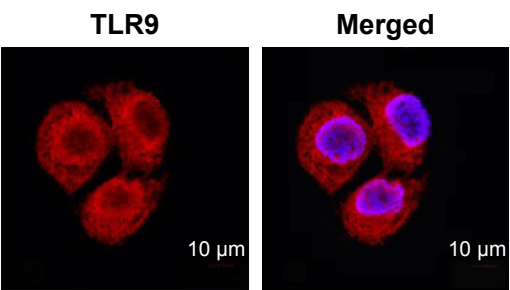

$43 \mathrm{kD}$
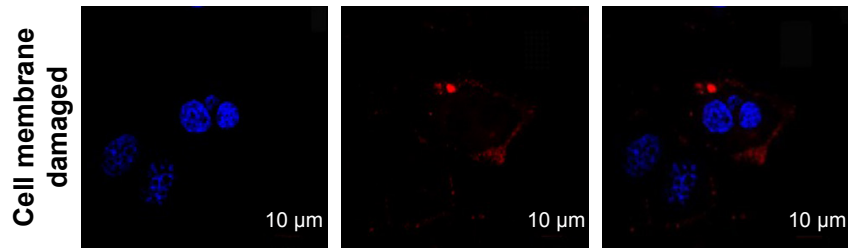

E

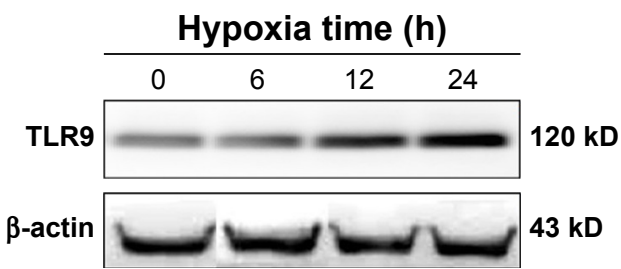

Figure 5 The expression of TLR9 in human ovarian tissue and SKOV3 cells.

Notes: (A) The expression levels of TLR9 and MRP in nine ovarian cancer tissues; (B) relevance between the TLR9 and MRP level in human ovarian cancer tissues; (C) TLR9 was expressed in SKOV3 cells by immunofluorescence; (D) effect of hypoxia on the mRNA level of TLR9 in the SKOV3; (E) effect of hypoxia on the protein level of TLR9 in the SKOV3. $* P<0.05$, compare with normoxia group (0 hour).

Abbreviations: TLR, toll-like receptor; MRP, multidrug resistance-associated protein; DAPI, 4',6-diamidino-2-phenylindole.

Additionally, only cell surface TLR9 plays an important role in cancer cell proliferation and progression. ${ }^{13}$ TLR9 expression was exhibited in esophageal cancer cells and was associated with cell proliferation and differentiation. However, mtDNA that escapes from autophagy leads to the expression of TLR9 in the cytoplasm, activating inflammatory response in cardiomyocytes and inducing myocarditis. ${ }^{14}$ Chemosensitivity of cisplatin-treated ovarian cancer cells was attenuated by CpG ODN and fMLF. Thus, activation of FPR and TLR9 in ovarian cancer cells is associated with chemoresistance of tumor cells.

Upregulated expression of MRP, P-glycoprotein (P-gp), p53 and Beclin-1 were observed in ovarian cancer cells with hypoxic conditions, which were inhibited after FPR antagonist treatment. Therefore, the upregulated expression of MRP, P-gp, p53 and Beclin-1 is closely connected with FPR activation. Besides, the upregulated the expression of MRP, P-gp and p53 were inhibited by TLR9 antagonist CQ, suggesting the involvement of TLR9mediated chemoresistance. It is reported that lapatinib sensitized breast cancer cells to doxorubicin by repressing MRP-1 expression induced by doxorubicin via PI3K/AKT and $\mathrm{p} 38 \mathrm{MAPK}$ signal pathways. ${ }^{15}$ MRP-4 is upregulated in hepatic encephalopathy via $\mathrm{p} 38 \mathrm{MAPK}$ signaling, which may promote cell proliferation. ${ }^{16}$ Some studies have shown that the expression of multidrug resistant $\mathrm{P}$-gp is associated with NF- $\mathrm{KB}$ signal pathway. P-gp is induced by cyclooxygenase-2 through NF- $\kappa B$, which is repressed by NF- $\kappa B$ pathway inhibitor pyrrolidine dithiocarbamate. ${ }^{17} \mathrm{In}$ addition, drug resistance of SGC7901/DDP cells to cisplatin 

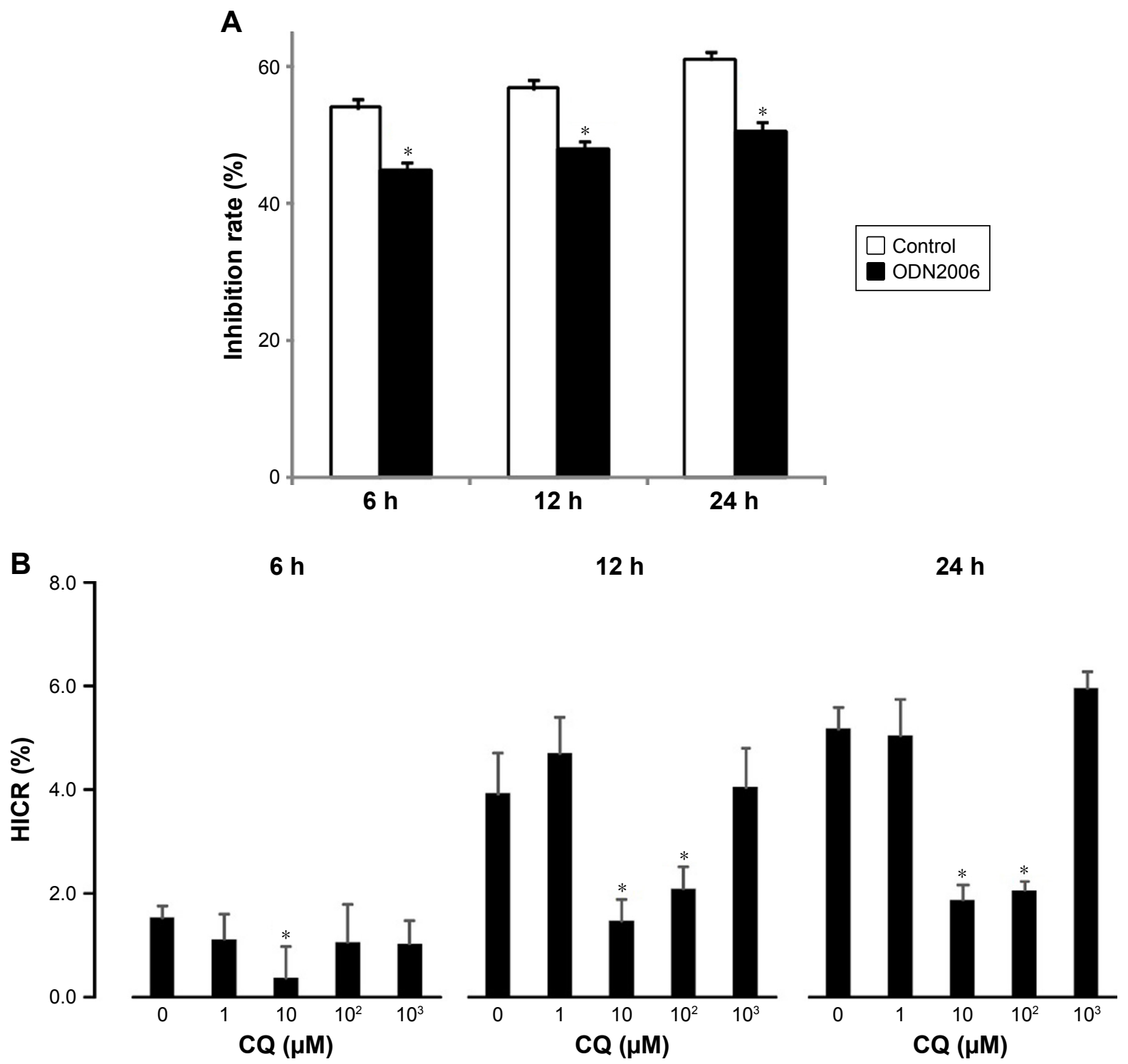

Figure 6 Effect of TLR9 to chemoresistance by hypoxia.

Notes: (A) TLR9 agonist CPG ODN2006 reduces the sensitivity of SKOV3 to cisplatin; (B) TLR9 antagonist attenuates hypoxia supernatant-induced cell resistance to cisplatin. $* P<0.01$, compared with control group.

Abbreviations: $\mathrm{HICR}$, hypoxia-induced chemoresistance; $\mathrm{CQ}$, chloroquine.

could be reversed by downregulating P-gp expression and upregulating apoptosis via downregulated NF- $\mathrm{KB}$. It is reported that tumor chemoresistance is connected with p53-mediated gene repair and p53 is upregulated by hypoxia resulting in drug resistance. ${ }^{18,19}$ Previous studies have shown that TLR9 regulates gene expression through PI3K/AKT, p38MAPK and NF- $\mathrm{KB}$ signal pathways. In our research, TLR9 plays an important role in HICR of ovarian cancer cells by upregulating MRP, P-gp and p53 via MAPK and NF- $\kappa B$ pathway. Autophagy also plays an important role in the development of tumor chemotherapy resistance, which is regulated by Beclin-1. ${ }^{20,21}$ Beclin- 1 is a specialized molecule which plays a multifunctional role in the cell. Beclin-1, by being directly involved in the initiation of autophagy, indirectly implicates an important role in numerous biological cellular processes, including development, endocytosis, adaptation to stress, aging and cell death. It is reported that Beclin-1 protein expression decreased in the drug-resistant ovarian cancer tissues. ${ }^{22}$ Our data showed that the expression of Beclin-1 was upregulated by hypoxia in ovarian cancer cells and this effect was reduced by tBoc, suggesting FPR plays a role in autophagy-mediated chemoresistance. But this is not contradictory. The effect of autophagy varies during different stimulation, which not 

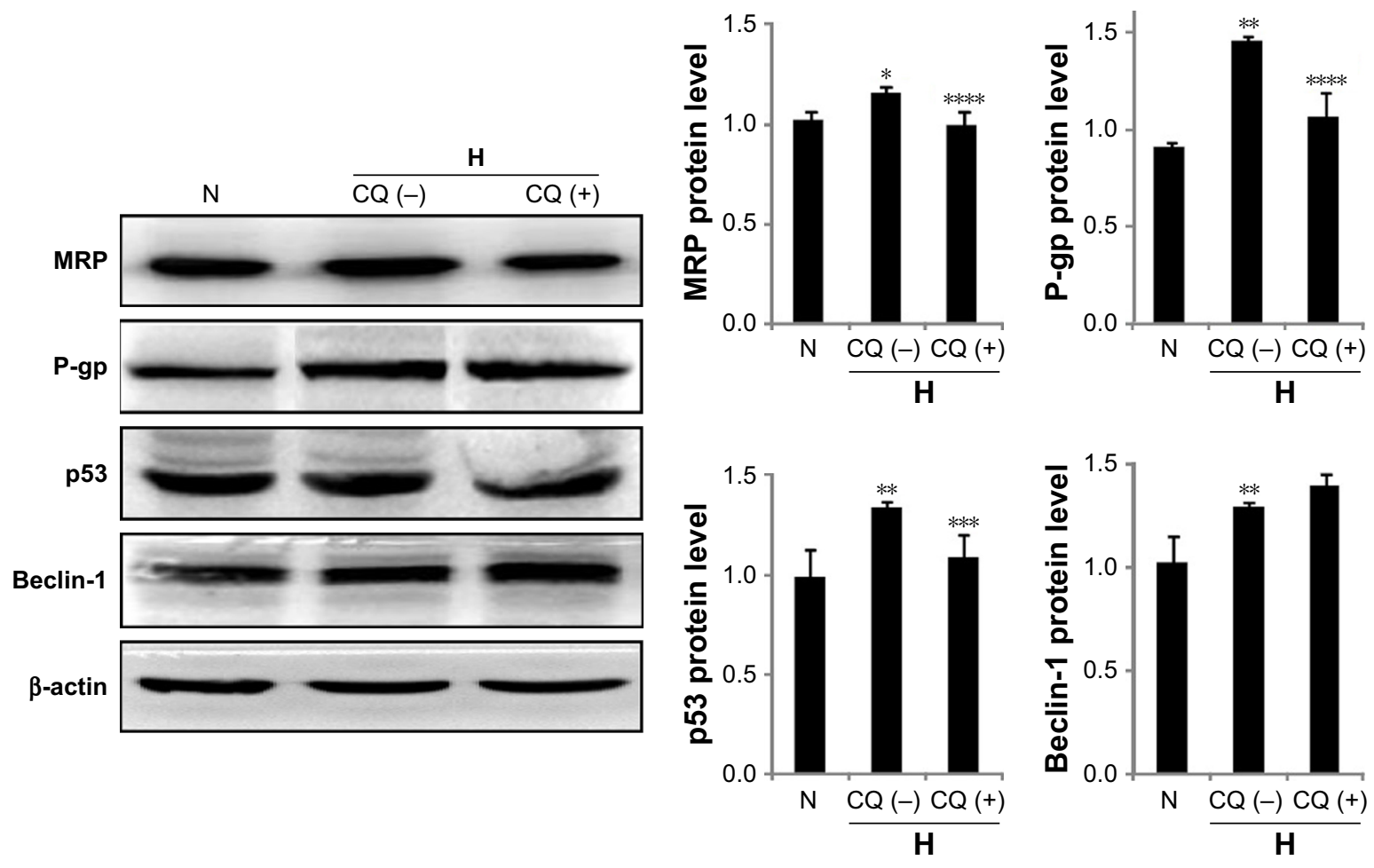

Figure 7 The expression of MRP, P-gP, p53 and Beclin-I in ovarian cancer cells with/without CQ pretreatment.

Notes: $* P<0.05, * * P<0.0$ I, compared with normoxia cell group; $* * * P<0.05$, $* * * * P<0.0$ I, compared with hypoxia without $C Q$ cell group.

Abbreviations: MRP, multidrug resistance-associated protein; $\mathrm{CQ}$, chloroquine.

only inhibits cancer progression but also eliminates necrotic organelles thus protecting cells. ${ }^{23,24}$

\section{Conclusion}

In summary, our data demonstrate that hypoxia-induced TLR9 and FPR expression causes the upregulation of MRP and reduction of chemosensitivity to cisplatin, resulting in chemotherapy resistance of ovarian cancer cells, suggesting FPR/ TLR9 as the potential targets for chemotherapy senitization. ${ }^{25}$

\section{Acknowledgments}

This study was funded by the grants from the National Natural Science Foundation of China (Grant No 81273608), research project of Chongqing Science and Technology (No cstc2015jcyjBX0018) and research project of Third Military Medical University (No 2015XZH19).

\section{Disclosure}

The authors report no conflicts of interest in this work.

\section{References}

1. Teicher BA. Hypoxia and drug resistance. Cancer Metastasis Rev. 1994;13(2):139-168.

2. Höckel M, Vaupel P. Tumor hypoxia: definitions and current clinical, biologic, and molecular aspects. J Natl Cancer Inst. 2001;93(4): 266-276.
3. Chen Z, Lai TC, Jan YH, et al. Hypoxia-responsive miRNAs target argonaute 1 to promote angiogenesis. J Clin Invest. 2013;123(3): 1057-1067.

4. Barton GM, Kagan JC, Medzhitov R. Intracellular localization of tolllike receptor 9 prevents recognition of self DNA but facilitates access to viral DNA. Nat Immunol. 2006;7(1):49-56.

5. Vaz J, Andersson R. Intervention on toll-like receptors in pancreatic cancer. World J Gastroenterol. 2014;20(19):5808-5817.

6. Babbin BA, Jesaitis AJ, Ivanov AI, et al. Formyl peptide receptor-1 activation enhances intestinal epithelial cell restitution through phosphatidylinositol 3-kinase-dependent activation of Rac1 and Cdc42. J Immunol. 2007;179(12):8112-8121.

7. Cheng TY, Wu MS, Lin JT, et al. Formyl peptide receptor 1 expression is associated with tumor progression and survival in gastric cancer. Anticancer Res. 2014;34(5):2223-2229.

8. Ye RD, Boulay F, Wang JM, et al. International Union of Basic and Clinical Pharmacology. LXXIII. Nomenclature for the formyl peptide receptor (FPR) family. Pharmacol Rev. 2009;61(2):119-161.

9. Bizzarro V, Belvedere R, Migliaro V, Romano E, Parente L, Petrella A. Hypoxia regulates ANXA1 expression to support prostate cancer cell invasion and aggressiveness. Cell Adh Migr. 2017;11(3):247-260.

10. Liu Y, Yan W, Tohme S, et al. Hypoxia induced HMGB1 and mitochondrial DNA interactions mediate tumor growth in hepatocellular carcinoma through toll-like receptor 9. J Hepatol. 2015;63(1):114-121.

11. Tohme S, Yazdani HO, Al-Khafaji AB, et al. Neutrophil extracellular traps promote the development and progression of liver metastases after surgical stress. Cancer Res. 2016;76(6):1367-1380.

12. Brahimi-Horn MC, Ben-Hail D, Ilie M, et al. Expression of a truncated active form of VDAC1 in lung cancer associates with hypoxic cell survival and correlates with progression to chemotherapy resistance. Cancer Res. 2012;72(8):2140-2150.

13. Tanaka J, Sugimoto K, Shiraki K, et al. Functional cell surface expression of toll-like receptor 9 promotes cell proliferation and survival in human hepatocellular carcinomas. Int J Oncol. 2010;37(4):805-814. 
14. Oka T, Hikoso S, Yamaguchi O, et al. Mitochondrial DNA that escapes from autophagy causes inflammation and heart failure. Nature. 2012;485(7397):251-255.

15. Kwon YS, Chun SY, Nam KS, Kim S. Lapatinib sensitizes quiescent MDA-MB-231 breast cancer cells to doxorubicin by inhibiting the expression of multidrug resistance-associated protein-1. Oncol Rep. 2015;34(2):884-890.

16. Jördens MS, Keitel V, Karababa A, et al. Multidrug resistanceassociated protein 4 expression in ammonia-treated cultured rat astrocytes and cerebral cortex of cirrhotic patients with hepatic encephalopathy. Glia. 2015;63(11):2092-2105.

17. Gu KS, Chen Y. Mechanism of P-glycoprotein expression in the SGC7901 human gastric adenocarcinoma cell line induced by cyclooxygenase-2. Asian Pac J Cancer Prev. 2012;13(5):2379-2383.

18. Gao JL, Shui YM, Jiang W, et al. Hypoxia pathway and hypoxia-mediated extensive extramedullary hematopoiesis are involved in ursolic acid's anti-metastatic effect in 4T1 tumor bearing mice. Oncotarget. 2016; 7(44):71802-71816.

19. Hirabayashi T, Takahashi H, Watanabe M, Tachibana T. Establishment and characterization of a squamous cell carcinoma cell line, designated $\mathrm{hZK}-1$, derived from a metastatic lymph node tumor of the tongue. Hum Cell. 2017;30(4):319-326.
20. Ishima Y, Inoue A, Fang J, et al. Poly-S-nitrosated human albumin enhances the antitumor and antimetastasis effect of bevacizumab, partly by inhibiting autophagy through the generation of nitric oxide. Cancer Sci. 2015;106(2):194-200.

21. Tan Q, Wang M, Yu M, et al. Role of autophagy as a survival mechanism for hypoxic cells in tumors. Neoplasia. 2016;18(6):347-355.

22. Ying H, Qu D, Liu C, et al. Chemoresistance is associated with Beclin-1 and PTEN expression in epithelial ovarian cancers. Oncol Lett. 2015;9(4):1759-1763.

23. Fu J, Shang HX, Jia HT. [Relationship of beclin1 with autophagy and tumor]. Sheng Li Ke Xue Jin Zhan. 2012;43(2):155-158.

24. Toton E, Lisiak N, Sawicka P, Rybczynska M. Beclin-1 and its role as a target for anticancer therapy. J Physiol Pharmacol. 2014;65(4): 459-467.

25. Mohamed FE, Al-Jehani RM, Minogue SS, et al. Effect of toll-like receptor 7 and 9 targeted therapy to prevent the development of hepatocellular carcinoma. Liver Int. 2015;35(3):1063-1076.

\section{Publish your work in this journal}

OncoTargets and Therapy is an international, peer-reviewed, open access journal focusing on the pathological basis of all cancers, potential targets for therapy and treatment protocols employed to improve the management of cancer patients. The journal also focuses on the impact of management programs and new therapeutic agents and protocols on

\section{Dovepress}

patient perspectives such as quality of life, adherence and satisfaction. The manuscript management system is completely online and includes a very quick and fair peer-review system, which is all easy to use. Visit http://www.dovepress.com/testimonials.php to read real quotes from published authors. 